Meta

Journal des traducteurs

Translators' Journal

\title{
Table ronde sur la fonction documentation dans les services linguistiques
}

\section{Rachel Boutin-Quesnel}

Volume 25, numéro 1, mars 1980

La documentation

URI : https://id.erudit.org/iderudit/003890ar

DOI : https://doi.org/10.7202/003890ar

Aller au sommaire du numéro

Éditeur(s)

Les Presses de l'Université de Montréal

ISSN

0026-0452 (imprimé)

1492-1421 (numérique)

Découvrir la revue

Citer cet article

Boutin-Quesnel, R. (1980). Table ronde sur la fonction documentation dans les services linguistiques. Meta, 25(1), 28-41. https://doi.org/10.7202/003890ar d'utilisation que vous pouvez consulter en ligne. 


\title{
Table ronde sur la fonction documentation dans les services linguistiques
}

\author{
RACHEL BOUTIN-QUESNEL \\ chef du Service linguistique, \\ Compagnie T. Eaton Limitée
}

Des professionnels de la documentation, de la terminologie et de la traduction se sont réunis pour discuter entre eux de la fonction documentation au sein de services linguistiques. Le programme de cette table ronde couvrait d'abord les particularités d'un centre de documentation à l'intérieur d'un service linguistique : ses objectifs, son organisation matérielle, les systèmes de classification utilisés et la nature de son fonds documentaire. En second lieu, il s'agissait de définir le rôle de chacun : documentalistes d'une part, traducteurs, rédacteurs et terminologues (les usagers) d'autre part; les attentes de chacun et la formation particulière des documentalistes au regard de la traduction, de la rédaction et de la terminologie et celle des professionnels de ces mêmes disciplines au regard de la documentation.

Ont participé à la table ronde :

Rachel Boutin-Quesnel (R.B.) ;

Gilles Breton, traducteur, CN/Air Canada (G.B.) ;

Francine Doray, recherchiste, Hydro-Québec (F.D.) ;

Noëlle Guilloton, terminologue, O.L.F. (N.G.);

Louise Sansregret, chef des Services linguistiques, Société d'énergie de la

Baie James (L.S.) ;

Alfred Thériault, documentaliste, C.I.L. (A.T.).

\section{PaRTicularités}

\subsection{Objectifs}

R.B. - Abordons la première partie de la discussion, soit les particularités d'un centre de documentation dans un service linguistique. Quels en sont d'abord les objectifs ?

N.G. - Il me semble d'abord qu'un tel centre doit répondre aux besoins proprement linguistiques de l'entreprise, si on l'oppose au service de documentation général ou bibliothèque centrale, s'il en existe une, qui répond à des besoins beaucoup plus variés. Le premier doit donc s'attacher à la langue générale et aux langues de spécialité et par conséquent accorder de l'importance à la qualité de la langue des documents, en plus de celle qu'il accorde à la qualité de l'information qu'ils contiennent. Un autre objectif, il me semble, de ce centre de documentation serait d'informer les traducteurs, terminologues et rédacteurs de ce qui se passe dans leur profession : associations, cours de recyclage éventuels, conférences, colloques, congrès, etc.

L.S. - Ce qui vient d'être dit résume bien les objectifs d'un centre de documentation de service linguistique. Je voudrais toutefois insister sur la différence fondamentale qui 
existe entre un tel centre et une bibliothèque centrale ou tout autre centre de documentation spécialisé. Dans ces derniers en particulier sont regroupés des ouvrages concernant principalement l'activité en cause ; il en est ainsi, il est vrai, dans un service linguistique, à l'exception cependant qu'on devra y trouver, en plus, un large éventail de documents portant sur d'autres domaines afin d'y puiser et les renseignements de fond et les formes linguistiques pertinentes. C'est pourquoi le documentaliste d'un service linguistique doit travailler en étroite collaboration avec les professionnels du service en ce qui a trait à la qualité des ouvrages, surtout sur le plan de la forme.

R.B. - La qualité de la forme est donc autant à rechercher que celle du fond ?

L.S. - Pour moi, c'est un des objectifs visés dans un service linguistique. C'est ce qui distingue particulièrement le centre de documentation d'un service linguistique, de tout autre centre de documentation. Il serait aussi souhaitable qu'il soit tenu compte de cette particularité à l'intérieur des études en documentation.

R.B. - Nous reviendrons sur ce dernier point. Pour vous, Gilles Breton, quel objectif viseriez-vous si vous aviez à mettre sur pied un centre de documentation?

G.B. - Ce serait d'abord d'inventorier constamment les besoins du service linguistique à desservir. Le documentaliste doit travailler en étroite collaboration avec le chef du service afin de pouvoir connaître un peu à l'avance les sujets pour lesquels il lui faudra trouver de la documentation comme, par exemple, les nombreux travaux en gestion informatisée qui touchent tous les secteurs de l'entreprise. Il doit donc être très conscient des besoins du service et même les prévoir.

R.B. - Par conséquent, un autre objectif d'un centre de documentation dans un service linguistique serait d'aller au-devant des besoins.

G.B. - Oui, parce qu'au rythme où on doit produire actuellement, il arrive souvent que la documentation ne parvienne qu'une fois le travail terminé. Dans bien des cas, une telle situation pourrait être évitée. Dès la réception d'une demande de traduction, le chef de service devrait en faire part au documentaliste qui pourrait immédiatement se mettre en chasse. De mon point de vue de traducteur c'est une question de planification.

R.B. - Et vous, Alfred Thériault...

A.T. - Chez nous la bibliothèque centrale est trop éloignée du service linguistique d'une part et, d'autre part, elle ne comporte que des ouvrages en anglais. Il nous fallait done établir un centre de documentation qui réponde aux besoins quotidiens du service. Actuellement 95 pour cent de notre documentation est en français. Nous cherchons principalement à avoir des ouvrages didactiques afin d'aider le traducteur à bien comprendre les sujets qu'il traite, car la plupart du temps il n'est ni spécialiste, ni ingénieur, ni chimiste, etc.

R.B. - Fournir aux usagers, dans un domaine donné, les éléments de connaissances techniques dont ils ont besoin pour mener à bien leur travail serait aussi un objectif du centre.

A.T. - En effet, mais il s'agit surtout de leur fournir les documents pertinents.

G.B. - Il y a un élément humain très important dans les échanges entre traducteurs et documentalistes. Ces derniers contribuent à la formation des premiers en leur apprenant à s'y retrouver dans un centre de documentation.

A.T. - En effet, on vient souvent voir le documentaliste pour une explication, ou pour savoir où trouver telle information. Il doit donc pouvoir répondre et en même temps expliquer à l'usager comment trouver ce qu'il cherche. Les traducteurs ainsi formés s'y retrouvent de mieux en mieux par eux-mêmes et n'ont recours au documentaliste 
qu'en dernier ressort. Mais il reste que le documentaliste doit faire en sorte que l'usager ait rapidement en main la documentation dont il a besoin.

R.B. - Un autre objectif du centre de documentation est donc d'aider le traducteur à trouver rapidement l'information pertinente.

F.D. - Une autre particularité d'un centre de documentation dans un service linguistique est la «personnalisation» du service rendu. Beaucoup plus qu'à la bibliothèque générale où les bibliothécaires ne connaissent pas nécessairement le contenu des documents qui s'y trouvent. Le documentaliste peut indiquer à son « client » où trouver le renseignement dont il a besoin. Ce dernier à son tour peut informer le documentaliste sur le contenu réel de l'ouvrage consulté. Il y a ainsi échange, participation.

N.G. - Le documentaliste va ainsi plus loin dans la recherche.

\subsection{Organisation matérielle}

R.B. - L'organisation matérielle d'un centre de documentation dans un service linguistique a-t-elle un caractère distinctif ?

F.D. - Elle est comme dans toute bibliothèque.

N.G. - Dans le cas d'une entreprise où il $\mathrm{y}$ a une bibliothèque centrale et un centre de documentation 《 linguistique $»$, adopte-t-on le même système, y a-t-il conflit ?

F.D. - À Hydro-Québec, la situation tend à s'uniformiser. La bibliothèque centrale s'occupe du catalogage et de la classification de notre documentation et en établit même les fiches bibliographiques. Par contre, nous préférons faire nous-mêmes les fiches-sujets qui doivent être en français et répondre aux besoins terminologiques.

N.G. - Est-ce qu'au CN, les ouvrages sont commandés par la bibliothèque centrale et, une fois arrivés, y sont-ils catalogués?

G.B. - Oui, pour la plupart des ouvrages. Les commandes passent par leur système. Ils établissent les fiches bibliographiques. Notre documentaliste s'occupe ensuite de classer le tout : documents et fiches. Il arrive parfois que nous achetions nous-mêmes un ouvrage dont nous avons un pressant besoin. La documentaliste le traite temporairement et l'envoie à la bibliothèque pour traitement définitif, une fois l'urgence passée. Mais c'est toujours très long de passer par la bibliothèque centrale.

N.G. - Ce procédé de traitement temporaire, quand il y a urgence, me semble aplanir certaines difficultés, surtout lorsqu'on ne peut prévoir les besoins longtemps à l'avance. Dans le cas où il $\mathrm{y}$ a une bibliothèque centrale, est-il possible d'avoir au centre de documentation un double du fichier de cette dernière?

F.D. - Il est actuellement projeté de microfilmer le fichier de la bibliothèque centrale et de l'avoir ainsi à notre disposition.

N.G. - Je me demande aussi s'il appartient à la Documentation de tenir un fichier des textes traduits dans le service, ou si cela revient plutôt à l'administration du service ; dans le but surtout d'éviter que les mêmes textes ne soient traduits deux fois, tels les rapports d'activité, les états financiers, etc.

F.D. - À Hydro-Québec, on a intégré au fichier documentation des fiches-sujets portant sur les travaux de traduction. Ces fiches sont éliminées, après 5 ans, à moins qu'il ne s'agisse de travaux importants ou encore d'actualité.

G.B. - Cela veut-il dire que vous intégrez la fonction «classement des traductions》 ou s'il ne s'agit que de repérage?

F.D. - De façon systématique tous les travaux importants de traduction sont sur les rayons : publications, rapports internes, contrats, etc. 
N.G. - Qu'en est-il des définitions d'emploi ou des descriptions de tâches, choses assez fastidieuses à traduire?

G.B. - Est-ce confié au centre de documentation ?

A.T. - A CIL on a établi un système de classement pour tous les travaux de traduction. Les traducteurs rédigent les fiches-sujets, lesquelles sont revues par les réviseurs. Puis les documents sont classés par numéro de bordereau de commande et la ou les fiches-sujets renvoient à ce numéro.

L.S. - Chez nous, à la Société d'énergie de la Baie James, ce classement relève de l'administration du service et il est très simple. Cependant je trouve intéressante l'idée de l'intégrer au classement documentaire.

\subsection{Systèmes de classification}

R.B. - Dans un centre de documentation de service linguistique, quels systèmes de classification utilise-t-on? Les classifications conventionnelles ou des classifications maison?

L.S. - Aux Services linguistiques de la SEBJ nous avons adopté le système de la Banque de terminologie de l'Université de Montréal, parce qu'il est simple et qu'il répond à nos besoins. Il facilite de beaucoup le repérage des documents. Par contre, à la bibliothèque centrale, on utilise le système Dewey. Ce dernier nous a semblé un peu compliqué pour un centre de documentation dans un service linguistique.

G.B. - $\mathrm{Au} \mathrm{CN}$, c'est le système Dewey qui est appliqué, tout comme à la bibliothèque centrale puisque notre centre de documentation est relié à cette dernière. Je ne connais pas particulièrement ce système, mais chez nous les ouvrages sont classés par domaines, grands domaines, super-grands domaines. Un tableau des numéros Dewey correspondant aux domaines nous renvoient aux rayons. On s'y retrouve donc assez bien. L'avantage que je verrais à l'utilisation d'un système conventionnel, c'est de pouvoir s'y retrouver dans les autres bibliothèques qui ont le même système.

R.B. - Cependant il n'y a pas qu'un seul système conventionnel. Il y en a au moins deux importants : le Dewey et le Library of Congress.

N.G. - Ce dernier système est surtout utilisé dans les bibliothèques universitaires.

F.D. - A Hydro-Québec les deux systèmes cohabitent présentement, mais on s'achemine vers l'utilisation unique du Library of Congress, puisque l'entreprise travaille avec des chercheurs, qu'elle fait des échanges avec des bibliothèques universitaires.

N.G. - Il est beaucoup plus simple de n'utiliser qu'un seul système dans une même entreprise. Il est vrai que le Dewey est compliqué, mais il en existe une édition abrégée; les décimales sont réduites. On peut rester au grand domaine de généralité supérieure sans aller dans tous les raffinements.

A.T. - Aux Services linguistiques de la CIL, nous avons aussi adopté le système BTUM à cause de sa simplicité. De plus, à ce moment-là, la Banque offrait ses services par terminal. Il y avait donc de grandes possibilités de collaboration. À la bibliothèque centrale, par contre, on utilise le système Dewey, mais toute la documentation $y$ est en anglais. Nos ouvrages y sont aussi codés, car les achats sont centralisés. Cependant, une fois que nous avons les ouvrages en main, nous les codons selon notre système. Comme nous sommes en milieu fermé, nous pouvons fonctionner de cette façon. Nous allons à la bibliothèque centrale lorsque nous avons besoin d'ouvrages en anglais pour compléter des fiches terminologiques.

L.S. - Est-ce que des personnes à l'extérieur de votre service consultent votre documentation? 
A.T. - Quelquefois. Surtout des francophones qui veulent consulter des dictionnaires.

R.B. - Et à l'Office de la langue française ?

N.G. - Jusqu'à il y a quelques mois, c'était la Classification décimale universelle. On est en train actuellement de procéder à la conversion au Dewey. Je ne peux malheureusement pas fournir d'explications sur ces choix. La CDU est une classification utilisée plutôt en Europe et pour des besoins documentaires. Peut-être veut-on maintenant se convertir au Dewey pour des raisons d'uniformisation avec les autres bibliothèques d'ici.

R.B. - A l'école de bibliothéconomie est enseigné le fonctionnement des différents systèmes de classification mais, compte tenu l'expérience que vous avez acquise dans des services linguistiques, trouvez-vous que ces systèmes conviennent aux besoins des traducteurs et surtout des terminologues?

N.G. - Dans la mesure où l'on en reste à l'utilisation manuelle de la documentation, que l'on se contente d'avoir facilement accès aux rayons, de bouquiner sur place, d'avoir le moins possible affaire au fichier, tous les systèmes à mon avis se valent. Le plus simple alors est que l'usager puisse s'y retrouver. Cependant, par cette façon de faire on risque souvent de passer à côté du document que l'on cherche. En effet, on ne peut que rarement donner une seule cote à un ouvrage. Peut-être ne le trouvera-t-on pas sur le rayon du sujet où on pense qu'il a été classé. C'est pourquoi, tout usager d'un centre de documentation doit pouvoir et vouloir avoir accès au fichier. Tout est question de formation... les traducteurs et les terminologues doivent posséder les éléments de base qui leur permettent de s'y retrouver dans tout système de classification. Un système maison bien structuré qui se tient debout fera tout aussi bien l'affaire qu'un système conventionnel plus sophistiqué.

R.B. - Quel système utilisait-on dans le cabinet de traduction où vous étiez avant d'entrer à l'OLF ?

N.G. - Là aussi on a adopté le système BTUM.

R.B. - Ce système semble en passe de devenir conventionnel!

L.S. - C'est en effet un système bien connu dans les bureaux de traduction.

A.T. - La simplicité de ce système permet beaucoup plus d'aller de mémoire sur les rayons. On va au fichier quand on ne se rappelle pas où trouver ce que l'on cherche.

F.D. - Cette habitude toutefois me semble très dangereuse.

N.G. - Beaucoup d'information se perd de cette façon, des documents restent ignorés, parce qu'on a des habitudes...

G.B. - Il faut que la documentation soit très bien dépouillée et bien codée, pour que toute information puisse être repérée facilement.

N.G. - Avec plusieurs cotes, tout ouvrage se retrace même s'il n'est logé qu'à un seul endroit. Le fichier permet de tout retrouver.

R.B. - C'est par le fichier qu'on peut aussi retracer l'information contenue dans les périodiques qu'on aura dépouillés.

G.B. - Il faut créer l'habitude du fichier; entre autres, inciter l'usager à vérifier le contenu du fichier en le tenant au courant de toutes nouvelles acquisitions.

L.S. - Malheureusement, tous les bureaux de traduction ne sont pas organisés au point d'avoir un fichier bibliographique. Il y a même des bureaux qui n'ont pas de documentation. Aussi y a-t-il encore beaucoup à faire pour susciter plus d'intérêt vis-à-vis de la documentation. 


\subsection{Nature du fonds documentaire}

R.B. - La particularité d'un centre de documentation dans un service linguistique ne concerne-t-elle pas, de plus, le type de documents qui doivent s'y trouver?

L.S. - Si l'on cerne bien l'activité de l'entreprise où se trouve ce centre de documentation, il va de soi que l'éventail des domaines à couvrir y sera conforme. Dans le cas de la SEBJ, il pourra s'étendre des relations de travail jusqu'aux négociations avec les Amérindiens en passant par la construction des barrages, le montage des lignes, la passation des contrats, etc.

N.G. - Dans toutes les entreprises on doit être bien documenté en gestion, en comptabilité, en finances.

G.B. - À cela, il faut ajouter tous les ouvrages généraux comme les encyclopédies, les dictionnaires de langue, etc.

L.S. - Il faut beaucoup d'ouvrages linguistiques.

N.G. - On y trouvera évidemment plus d'ouvrages en français qu'en anglais.

R.B. - Cependant, il ne faut pas négliger les ouvrages de langue anglaise qui nous aident à bien cerner les notions que nous avons à traiter.

N.G. - Surtout là où il se fait de la terminologie bilingue, les ouvrages doivent être dans les deux langues, mais avec un fort pourcentage d'ouvrages français.

L.S. - En ce qui concerne les normes, il est très utile d'avoir celles de l'AFNOR plutôt que celles de l'ACNOR. On retrouve davantage ces dernières dans les bibliothèques d'entreprise.

R.B. - Les normes ACNOR sont utiles, pour les données techniques qu'elles fournissent, tandis que celles de l'AFNOR le sont aussi pour la qualité de la forme.

L.S. - C'est là que se situe la difficulté pour un documentaliste dans la sélection de la documentation. Il lui faut travailler en étroite collaboration avec sa «clientèle» afin de bien saisir ses besoins, à la fois techniques et linguistiques.

N.G. - J'aimerais insister aussi sur l'accent qu'on doit mettre sur l'actualité linguistique de la documentation. Le service doit vraiment être au fait de tout ce qui se passe dans le domaine linguistique. Je pense aux chroniques de langue dans les journaux, aux lexiques dans les revues professionnelles ou de langue, etc.

A.T. - Ce doit être la même chose dans les domaines techniques qui nous intéressent ; il faut se tenir à jour.

N.G. - Mais on peut trouver cette information dans une bibliothèque centrale.

R.B. - Oui, mais elle risque fort d'être surtout en anglais.

A.T. - L'information des revues est toujours plus à jour que celle des dictionnaires et des monographies. C'est ce qui nous incite à faire le dépouillement des revues.

F.D. - Malheureusement, il arrive souvent qu'on n'a pas le temps de les dépouiller ou qu'on ne prend pas le temps de le faire.

A.T. - En certains endroits, on essaie de mettre les traducteurs dans le coup, mais c'est assez difficile. Moi, je fais ce dépouillement et j'en établis le répertoire, un peu à la façon du système KWICK, avec un index.

F.D. - Va pour le dépouillement documentaire, mais le dépouillement terminologique ?

A.T. - Cela revient au terminologue. 
F.D. - A Hydro-Québec, nous faisons les deux.

A.T. - Pour moi, le dépouillement terminologique des revues est une attribution de la Terminologie et non de la Documentation.

R.B. - Indépendamment des listes ou répertoires des articles retenus, établit-on une fiche bibliographique pour chacun de ces articles?

F.D. - On en fait encore, mais de moins en moins; car elles servent plus ou moins ; au bout de cinq ans, l'article est périmé et la fiche est encore dans le fichier.

A.T. - Nous n'en faisons pas. Nous faisons un nettoyage annuel de nos périodiques pour ne retenir que certains articles particulièrement intéressants. Le répertoire demeurant, tout article qui y est inscrit peut être récupéré chez Informatech France-Québec ou à la Bibliothèque nationale.

R.B. - $\AA$ Hydro-Québec, que faites-vous des articles récents ?

F.D. - On les met dans des boîtes dites d'archives. Une fiche générale indique "énergie solaire " ou encore " énergie éolienne », voir boîte $n^{\circ} \ldots$ On y fait le ménage périodiquement. Autrement, nous aurions trop de fiches inutiles dans notre fichier, la technique évoluant si rapidement.

\section{RÔLES, ATTENTES ET FORMATION}

\subsection{Rôle du documentaliste}

R.B. - Dans cette deuxième partie nous discuterons du rôle de chacun, de ses attentes en ce qui concerne la documentation et de la formation du documentaliste dans un service linguistique. En premier lieu, quel est le rôle du documentaliste?

A.T. - En plus de tout l'aspect purement technique de la tenue des documents et de l'enrichissement du fonds documentaire, mon rôle consiste à m'assurer qu'on utilise à fond la documentation que nous avons, à renseigner la clientèle sur les richesses cachées de la documentation et à lui en faciliter l'accès ainsi qu'à prévoir, dans la mesure du possible, les besoins en documentation. Je dois aussi chercher de nouvelles méthodes d'accès à l'information. Il $\mathrm{y}$ a de plus les relations avec les autres centres de documentation, les échanges de services.

T.D. - Chez nous, c'est différent car je ne suis pas seule. Une autre documentaliste s'occupe de l'organisation matérielle du centre, des acquisitions, de la gestion des périodiques, de la tenue du fichier bibliographique, du bulletin d'information interne. Quant à.moi, j'effectue des recherches documentaires pour les traducteurs et les terminologues, ce qui occupe la plus grande partie de mon temps. Je participe plutôt à la recherche terminologique. $J e$ fais aussi du dépouillement dans les revues terminologiques et générales. Je participe également à la gestion du fichier terminologique.

N.G. - Est-ce que chez vous on s'occupe de l'initiation des membres du service à la documentation?

F.D. - Oui. Dès son arrivée au service, chaque rédacteur-traducteur fait une visite de la documentation. On lui en fait faire un tour assez rapide. On lui montre les fichiers, on lui indique les principaux ouvrages de référence. Après quelques jours de travail, il est prêt à recevoir de plus amples explications. Et l'initiation se poursuit tant que - l'usager n'a pas parfaitement saisi le fonctionnement du centre de documentation.

N.G. - Je voudrais aussi souligner l'importance du rôle de liaison que le documentaliste joue entre son service et les autres sources documentaires de l'entreprise et de l'extérieur. C'est lui qui reçoit ou doit recevoir les divers bulletins et en extraire ce qui est intéressant. C'est à lui qu'on s'adresse quand on veut des renseignements sur tel organisme. 
F.D. - Le documentaliste doit être au courant des services que peuvent offrir d'autres entreprises. On ne peut pas avoir toutes les sources.

N.G. - S'échange-t-on des listes d'acquisitions ?

A.T. - Je reçois d'I.B.M. leur liste d'acquisitions.

N.G. - Quand je travaillais dans le milieu de l'urbanisme, ça se faisait aussi. On était ainsi au courant de tout document ou article important qui paraissait et on savait qui l'avait.

L.S. - Je pense qu'il y a beaucoup d'échanges entre les services de traduction. Il serait à recommander qu'il y en ait aussi entre documentalistes.

N.G. - C'est justement ce que je me demande. Est-ce qu'il y a vraiment des échanges ou est-ce que chacun ne veut pas plutôt garder tout pour soi ?

L.S. - Il n'y a presque pas de documentalistes.

G.B. - Puisque cette fonction est récente, ne serait-il pas bon de lui intégrer dès maintenant cette tâche? Je n'insisterai jamais assez sur le rôle d'information du documentaliste. Il est la source de renseignements, il est l'âme du centre de documentation. Chez nous, la documentaliste produit un bulletin d'information très pertinent : acquisitions, rappel de certains trésors " cachés 》, appréciations d'ouvrages, extraits des journaux, etc. Certains services linguistiques le reçoivent aussi. On a toujours quelque renseignement à demander à la documentaliste ; c'est vraiment une personne clé.

R.B. - Le documentaliste a donc un rôle d'information ?

L.S. - D'information et de diffusion.

N.G. - J'insiste aussi sur le rôle d'initiation des usagers et sur celui de liaison avec les autres bibliothèques.

L.S. - Ce qui m'intéresse beaucoup dans le travail d'un documentaliste, c'est l'étroite collaboration qui s'établit entre lui et les terminologues. La collaboration documentaliste - traducteur ne se situe pas au même niveau. Elle concerne plutôt les critères d'utilisation, tandis qu'avec le terminologue, le documentaliste peut définir les critères de choix et de qualité des ouvrages.

\subsection{Rôle des usagers}

R.B. - Par conséquent, les usagers (traducteurs, terminologues et rédacteurs) ont un rôle à jouer en documentation.

A.T. - Les terminologues peuvent fournir de précieuses indications sur la qualité linguistique d'un ouvrage. Les documentalistes comptent aussi sur les terminologues et les traducteurs pour leur indiquer les ouvrages que ceux-ci ont découverts ici ou là.

L.S. - Il n'y a que les usagers qui peuvent savoir si un ouvrage est bien fait ou non. Sa qualité, bonne ou mauvaise, ne se découvre qu'à l'usage. On doit alors signaler ces faits au documentaliste.

G.B. - Le traducteur peut aussi aider à compléter le fichier-sujet à partir d'articles de revues ou de textes. Je pense aussi que le documentaliste a besoin de savoir dans quelle mesure servent les articles qu'il intègre au fichier. C'est une forme d'appréciation de son travail. Les traducteurs et les terminologues doivent aussi informer le documentaliste sur ce qu'ils font, de ce qui se fait dans l'entreprise.

N.G. - Il est important pour ce dernier d'avoir un assez bon profil de l'activité de chacun, afin de pouvoir leur signaler à l'occasion la documentation qui pourrait les intéresser. 
F.D. - Il est très important de savoir ce que traducteurs et terminologues font, de connaître les spécialisations de chacun, car à ce moment-là on sait qui aller voir lorsqu'on a certains problèmes documentaires.

N.G. - Il y a donc là un rôle de liaison entre les spécialistes.

G.B. - Le traducteur peut contribuer au travail du documentaliste, en s'assurant aussi que les textes qu'il a traduits et qui sont par la suite imprimés, sont versés au fonds documentaire, au lieu de n'apparaître que dans les dossiers de traduction.

N.G. - Pour ce qui est du dépouillement des périodiques, le documentaliste ne pourrait-il pas en confier un ou deux à chaque professionnel du service?

A.T. - On a déjà tenté l'expérience chez nous, mais cela n'a pas été un succès.

N.G. - Il y a des périodiques qui ne paraissent que quatre fois l'an. Ce n'est pas alors un gros travail.

L.S. - Il ne faut pas compter sur les traducteurs ou les rédacteurs pour faire le dépouillement des revues.

G.B. - Il faudrait partager le travail. Nous pourrions peut-être faire un dépouillement d'ordre terminologique. Le traducteur est sans doute celui qui est le plus au courant des besoins de l'entreprise.

L.S. - De façon générale qui doit faire le dépouillement terminologique ?

R.B. - Le terminologue.

A.T. - Ça pourrait être fait par le traducteur et vérifié par le terminologue.

R.B. - Pour ma part, je ne suis pas portée à confier le dépouillement terminologique à la Documentation. C'est plutôt le rôle de la Terminologie. Par contre, il revient à la Documentation de faire le dépouillement documentaire des périodiques.

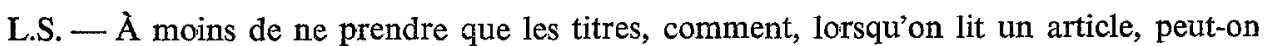
ne pas relever en même temps la terminologie qui s'y trouve?

A.T. - Moi quand je lis, je souligne les termes qui me semblent intéressants.

L.S. - Qu'est-ce que vous en faites ensuite?

A.T. - Je code l'article et l'intègre au répertoire. C'est ensuite au traducteur ou au terminologue de traiter cette terminologie.

G.B. - Ce type de dépouillement terminologique ne conduit pas nécessairement à l'établissement de fiches bilingues.

N.G. - Vous ne faites pas de résumé analytique du document? Un bref résumé et quelques mots clés?

F.D. - Non.

A.T. - Le répertoire en lui-même comporte des résumés, d'une certaine façon. Les titres KWICK comportent des mots clés. Si le titre de l'article n'est pas significatif, je puise dans le texte même les mots qui guideront le terminologue vers le contenu de l'article.

N.G. - Je verrais cette tâche partagée entre le documentaliste puis les traducteurs ou rédacteurs et les terminologues qui, eux, peuvent juger de l'utilité de tel article pour la traduction et la terminologie, de la qualité de la langue et de son apport terminologique.

R.B. - Ce travail pourrait être supervisé par le documentaliste, mais réparti entre les usagers. 
G.B. - Il faudrait toutefois que ce soit systématique, une demi-heure par jour, par exemple...

N.G. - ... ou une demi-journée par semaine. De toute façon, c'est excellent de lire en français quand on fait de la traduction, surtout quand on a en main une matière première intéressante, même si elle n'a aucun rapport direct avec le texte qu'on traduit à ce moment-là.

L.S. - Je pense qu'il serait difficile de systématiser cette pratique à heure fixe. C'est plutôt au moment où un traducteur ou un rédacteur termine un travail qu'il doit lire quelque peu avant d'entreprendre un autre travail. Il faut que le traducteur ou le rédacteur organise son temps pour pouvoir lire. C'est indispensable; autrement la qualité de leurs textes s'en ressent.

R.B. - Donc, si on incluait dans les fonctions du traducteur, du terminologue et du rédacteur la tâche de dépouiller un périodique en particulier, ils devraient pouvoir le faire.

\subsection{Attentes des usagers}

R.B. - Vous, Gilles, en tant que traducteur, qu'est-ce que vous attendez du documentaliste?

G.B. - D'abord je dois dire que notre documentaliste est épatante. Ce que j'attends d'elle, elle le fait déjà. Il y a d'abord le fichier-sujets qui est au point et qu'elle met continuellement à jour. Si je réussis à trouver ce dont j'ai besoin dans ce fichier, je n'ai pas besoin d'aller la voir.

R.B. - Supposons que vous ne trouviez rien au fichier, vous allez voir la documentaliste et qu'est-ce que vous voulez qu'elle fasse pour vous à ce moment-là ?

G.B. - Si, par exemple, je traduis un texte sur les moteurs Diesel, je m'attends à ce qu'elle téléphone ou écrive aux compagnies qui fabriquent ces moteurs, pour que ces dernières nous fournissent des notices techniques, tout au moins en anglais si elles n'en ont pas en français. Je ne connais évidemment pas toutes ses ressources, c'est pourquoi je l'informe de tout travail que j'ai à faire.

R.B. - Vous vous attendez à ce qu'elle fasse des démarches.

G.B. - Oui. De plus, comme je l'informe de ma spécialisation, je m'attends à ce qu'elle m'informe de toute documentation, surtout en français, relative à cette spécialisation. C'est un échange continu.

R.B. - Et vous Noëlle, comme terminologue, qu'est-ce que vous attendez du documentaliste?

N.G. - Je m'attends à ce qu'il fasse des démarches pour moi, qu'il ait acquis en temps voulu toute la documentation nécessaire qui a paru sur tel ou tel sujet.

R.B. - Supposons que votre travail soit assez court et la documentation sur le sujet pratiquement inexistante, est-ce que vous vous attendez à ce qu'il en cherche, même au risque que les documents n'arrivent pas à temps ?

N.G. - Oui, car si cette documentation ne sert pas dans l'immédiat, elle pourra servir plus tard. En plus de cette capacité de faire des démarches « documentaires», je m'attends aussi à ce que le documentaliste ait beaucoup de contacts, de personnesressources qui puissent suppléer à la documentation qu'il n'a pas, vers lesqueis il puisse m'aiguiller en cas de panne. Je pense que le documentaliste devrait avoir son petit réseau de "dépanneurs». 
G.B. - Les terminologues, pour leur part, ont leur réseau d'information, tout comme nous, traducteurs, avons le nôtre surtout au sein même de l'entreprise. Les documentalistes ont-ils un tel réseau dans l'entreprise même?

F.D. - Oui; il y a les personnes que nous connaissons et aussi l'annuaire de la compagnie...

N.G. - Le documentaliste nous éclaire aussi au moyen de répertoires de bibliothèques spécialisées, de centres de recherches divers, que les terminologues ne connaissent pas ou qu'ils ne savent pas comment contacter.

L.S. - Même si on travaille dans le domaine depuis longtemps, il y a beaucoup de ressources qu'on ne connaît pas.

R.B. - Indépendamment de la documentation à chercher à l'extérieur, est-ce que l'usager d'un centre de documentation au sein d'un service linguistique s'attend à ce que le documentaliste cherche pour lui le matériel documentaire dont il aura besoin pour exécuter un travail donné ? Est-ce qu'il s'attend à ce que-le documentaliste lui indique quel type de documentation consulter et même, quel document en particulier ? qu'il le mette directement sur la piste?

G.B. - Oui bien sûr ! Parce que d'une consultation à l'autre, le documentaliste enrichit sa propre connaissance du centre de documentation. Il peut arriver que tel ou tel ouvrage traitant du sujet qui nous intéresse, ne nous fournisse pas l'information que nous cherchons. Le documentaliste peut alors continuer la recherche et trouver autre chose.

R.B. - Est-ce qu'on doit s'attendre, par conséquent, à ce qu'il connaisse le contenu des ouvrages?

N.G. - Oui. Et à la limite, le documentaliste pourrait nous apporter toute la pile des documents pertinents sur notre bureau.

G.B. - Ça se fait, même si ça s'échelonne sur plusieurs jours, car souvent elle apporte ses trouvailles au fur et à mesure. Notre documentaliste est très sensibilisée au travail des traducteurs. C'est à nous par la suite d'analyser cette documentation et de prendre les décisions qui s'imposent, soit sur le plan connaissance du sujet, soit sur le plan linguistique.

\subsection{Attentes du documentaliste}

R.B. - Si maintenant, on demandait aux documentalistes ce qu'ils attendent des traducteurs, des rédacteurs et des terminologues.

N.G. - Un documentaliste s'attend à ce qu'on le tienne parfaitement au courant des projets en cours et de ceux qui sont à venir. Qu'on prenne le temps de lui expliquer en quoi consiste le travail, de la façon la plus détaillée possible.

F.D. - Le documentaliste s'attend aussi à recevoir de la rétroaction afin de savoir si les documents qu'il a fournis ont été utiles. L'usager peut fournir de précieuses informations sur tel ou tel document qui, par la suite, peuvent servir au documentaliste quand vient le moment de recommander ce même document.

N.G. - Les usagers doivent aussi signaler au documentaliste les ouvrages qui contribueront à enrichir le fonds documentaire.

R.B. - Mais est-ce que de son côté, le documentaliste ne devrait pas, de sa propre initiative, lire les bibliographies souvent présentées à la fin d'un article dans un périodique, les analyser et contribuer ainsi à enrichir la collection ?

N.G. - Normalement oui. Souvent le documentaliste fait circuler des titres d'ouvrages recueillis dans des bibliographies ou des photocopies de comptes rendus d'ouvrages et il 
s'attend à ce que trađucteurs, terminologues et rédacteurs disent ce qu'ils en pensent et indiquent quels sont les ouvrages qu'ils aimeraient voir acquérir par le centre de documentation.

Il y a aussi quelque chose de très utile. C'est de photocopier le sommaire des périodiques auxquels le centre est abonné et de le faire circuler parmi les usagers. Ainsi ces derniers peuvent repérer rapidement l'article qui les intéresse et ne lire que celui-là au moment où ils le pourront.

F.D. - Chez nous, à la bibliothèque centrale, il $\mathrm{y}$ a un bulletin mensuel dans lequel sont listés des titres d'articles.

N.G. - Il est plus intéressant de lire une photocopie de sommaire, qui attire le regard, que le caractère uniforme dactylographié. On voit tout de suite le format du périodique ; c'est moins rébarbatif à lire qu'une entrée bibliographique traditionnelle et ça fournit quand même des indications sur la longueur de l'article, l'auteur, etc. C'est vite parcouru, et pour les périodiques en particulier qui souvent ne sont pas assez utilisés, c'est un moyen simple et rapide de les rappeler à l'attention des usagers. Sans oublier que la photocopie est très importante pour ceux qui ont une mémoire visuelle. Il est évident qu'à ce moment-là, le documentaliste s'attend à ce que ces photocopies soient lues et rendent service...

\subsection{Formation}

R.B. - Si on abordait maintenant le dernier point de notre table ronde : quel genre dë formation devrait recevoir d'une part le documentaliste qui s'en va dans un service linguistique et d'autre part, le terminologue, le traducteur et le rédacteur par rapport à la documentation. Je pense que du côté de ces derniers beaucoup de choses se font; des programmes de perfectionnement sont même en cours. Mais du côté des documentalistes, la question ne se pose-t-elle pas encore dans son entier?

F.D. - Le minimum, selon moi, qu'on puisse exiger est un diplôme collégial en techniques de la documentation afin d'acquérir une bonne connaissance de la documentation, savoir comment l'organiser, quels sont les principaux ouvrages à consulter. Il y aurait avantage aussi à suivre des cours de spécialité, afin de posséder des notions essentielles de droit, par exemple, d'économique, etc., d'en connaître le jargon, d'avoir une vue plus large des problèmes de fonctionnement de cette spécialité, afin d'être plus apte à fournir une meilleure information documentaire.

R.B. - Cette formation qu'on dispense dans les cégeps prépare-t-elle les étudiants à travailler dans des centres de documentation de façon générale seulement, ou si elle leur fournit un petit quelque chose qui leur fait comprendre la nature et les besoins d'un service linguistique?

F.D. - Elle les prépare à travailler dans un centre de documentation de façon générale. Il n'y a pas de formation particulière pour les services linguistiques. Cette dernière s'acquiert plutôt en cours d'emploi. C'est à la personne qui recrute de vérifier si le candidat porte quelque intérêt aux questions linguistiques.

L.S. - Je pense que l'objet des études en bibliothéconomie est plutôt centré sur la façon de trouver un document que sur son aspect linguistique. Mais le programme d'études est déjà tellement chargé qu'il ne doit pas y avoir de place pour une option relative aux services linguistiques. Peut-être que si les débouchés étaient plus nombreux, il en serait autrement.

F.D. - Il n'y a même pas de débouchés pour les bibliotechniciens, les emplois sont rares.

N.G. - Selon moi, en plus d'un diplôme en techniques de la documentation, le candidat doit posséder une excellente culture générale, une réelle curiosité pour les faits de langue et une certaine capacité d'apprécier la valeur linguistique d'un ouvrage. 
Au cours des études universitaires de maîtrise (elles ne se situent qu'à ce niveau en bibliothéconomie), on pénètre dans des domaines plus spécialisés. On étudie les bibliographies de ces domaines. L'idéal serait peut-être d'obtenir d'abord un baccalauréat en linguistique ou en traduction, puis une maîtrise en bibliothéconomie. Cela en vaudrait sûrement la peine au point de vue efficacité.

R.B. - Et vous, Francine, qui êtes maintenant dans un service linguistique, comment êtes-vous arrivée à avoir cette sensibilité « linguistique 》?

F.D. - J'ai toujours aimé la lecture et je me suis toujours intéressée à l'utilisation correcte des mots.

G.B. - Le contact continuel avec l'essence même d'un service linguistique : traduction, rédaction et terminologie, éveille la curiosité des spécialistes de la documentation en poste ; ce qui les amène à s'intéresser davantage à ce que font les traducteurs, les rédacteurs et les terminologues.

R.B. - Il n'en reste pas moins qu'il est difficile de trouver des spécialistes de la documentation qui connaissent les besoins des services linguistiques.

G.B. - N'existe-t-il pas pour eux des examens de sélection, comme il en existe pour les autres professionnels de services linguistiques.

F.D. - Il peut en exister sur la capacité de trouver de la documentation. En général les candidats connaissent les fichiers-sujets. Il suffit de leur soumettre un problème documentaire et si le fichier est bien fait, ils trouveront ce qui existe dans le centre sur ce sujet. Cependant, cela ne veut pas dire qu'ils connaissent la documentation sur le plan linguistique ni qu'ils sont en mesure de comprendre l'aspect linguistique des demandes documentaires des usagers. C'est à l'expérience que ces choses s'apprennent.

N.G. - De plus, pour embaucher un documentaliste je pense qu'il est plus important que le candidat ait un intérêt marqué pour la langue plutôt que pour la spécialité même de l'entreprise.

L.S. - Selon moi, le programme d'études n'étant pas axé sur cet aspect linguistique, le candidat doit en revanche manifester une grande curiosité intellectuelle.

R.B. - N'y aurait-il pas moyen de donner un aperçu de ces besoins d'un service linguistique pendant la formation des spécialistes de la documentation, soit dans les cégeps, soit à l'université ? On doit sûrement indiquer aux étudiants les différents types de centres de documentation ainsi que les particularités et les objets de chacun d'eux. Pourquoi ne pas leur parler des centres de documentation propres aux services linguistiques? Ils existent au même titre que les autres.

G.B. - Les maisons d'enseignement ne sont-elles pas au courant de l'existence des centres de documentation dans les services linguistiques? Des étudiants pourraient y faire des stages. Est-ce qu'on vous a présenté cette possibilité d'aller travailler dans un service linguistique?

F.D. - Non, pas au cégep.

L.S. - Je me demande si les centres de documentation offrent des possibilités de stage?

F.D. - Les étudiants en techniques de la documentation ont des stages, mais ce sont surtout des stages techniques où ils peuvent montrer qu'ils savent faire du catalogage, etc.

R.B. - Mais les documentalistes proprement dits, où acquièrent-ils leur formation ?

F.D. - Formation de bibliotechnicien au cégep ou de bibliothécaire à l'université, seulement. 
N.G. - On s'appelle documentaliste quand le hasard fait qu'on travaille dans un centre de documentation.

R.B. - Il n'y a donc pas de formation de documentaliste en tant que telle ?

F.D. - Non, ça n'existe pas encore au Canada, mais ça existe en France.

R.B. - Et vous Alfred, comment avez-vous acquis votre formation?

A.T. - En cours d'emploi. J'ai d'abord été un traducteur en formation, mais très intéressé par la documentation. Comme on avait besoin d'un documentaliste dans notre service, j'ai accepté l'emploi.

R.B. - Vous étiez déjà sensibilisé à la traduction.

A.T. - Je savais quoi chercher.

L.S. - Vous deux, est-ce que vous aimez être documentalistes ?

A.T. - Oui, parce que j'aime lire. Je lis toutes les revues qui passent avec beaucoup de curiosité. Mon travail est très varié. Je m'occupe des acquisitions, de la codification, du classement, du dépouillement. Je fais beaucoup de consultations. Je connais le contenu de la documentation.

F.D. - Moi, j’aime beaucoup l'aspect a recherche» et l'aspect "langue» de ma fonction.

R.B. - Et c'est cette même préoccupation de recherche de la qualité linguistique qui rapproche traducteurs, rédacteurs, terminologues et documentaliste dans un service linguistique.

\section{CONCLUSION}

Il serait donc important que le documentaliste « linguistique » saisisse bien qu'il n'y a pas que les techniques de pointe qui intéressent sa clientèle, mais aussi les techniques de base ou courantes. Non seulement les derniers développements dans les domaines qui l'intéressent, mais aussi les activités en cours. Car à l'information technique, à tous les niveaux, s'ajoute l'expression langagière comme objet de recherche des usagers du centre de documentation.

Par conséquent, comme on commence à donner des cours d'initiation à la documentation aux traducteurs et surtout aux terminologues (la documentation étant l'outil essentiel de leur travail), ne pourrait-on pas souhaiter que des cours d'initiation à la traduction et surtout à la terminologie soient offerts à des documentalistes ? 